\title{
A CONDITION FOR FINITE DIMENSIONAL CONVEXITY
}

D. G. BOURGIN ${ }^{1}$

This note is devoted to a demonstration of

TheOREM. Let $K$ be a compact contractible subset of $R^{N}$. Suppose every support set is acyclic. Then $K$ is convex. The converse is obvious.

Here $R^{N}$ is $N$-dimensional Euclidean space. We use Cech homology with integer coefficient group [1]. Plane will be used for $N-1$ dimensional hyperplane. A support plane, $P$, is a plane meeting $K$ where $K$ is in just one of the two closed half spaces determined by $P . K \cap P$ is the support set for $P$. A weaker theorem, requiring the added hypothesis of local contractibility for $K$ was proved by Kuhn [2] who pointed out this hypothesis was essential for any proof based on his methods. Liberman [3] has established a slightly weaker theorem requiring contractibility for the support sets as the culmination of a long chain of subsidiary results.

Proof. We need $R^{n}$, the linear extension of $K$, so $n \leqq N$. To avoid triviality we assume $n>1$. Let $S^{n-1}$ and $Y$ refer to the $n-1$ topological sphere and to the metric unit $n-1$ sphere respectively. Each point $y$ in $Y$ determines a unique supporting hyperplane $P_{y}$ whose normals pointing away from $K$ are parallel translates in $R^{n}$ of the vector oy. We write $X$ for the set of boundary points of $K$ and $X_{y}$ for the support set $P_{y} \cap X$.

Let

$$
\Gamma=\left\{(y, x) \mid x \in X_{y}\right\} \subset Y \times X
$$

For each $x$ we introduce a subset of $Y$,

$$
Y_{x}=\{y \mid(y, x) \in \Gamma\} \text {. }
$$

Let $p$ and $q$ project $\Gamma$ into $Y$ and into $X$ respectively. Thus

$$
\begin{aligned}
& p: y \times X_{y}=y . \\
& q: Y_{x} \times x=x .
\end{aligned}
$$

The image of $\Gamma$ under $p$ covers $Y$ while that under $q$ is $X^{\prime} \subset X$. Obviously $X$ and $X^{\prime}$ are closed in $R^{n}$. Let $\hat{K}$ be the convex hull of $K$ and denote its boundary by $\hat{K} \cdot$. It is important to note that:

(a)

$$
X^{\prime} \subset \hat{K} \cdot \text {. }
$$

Received by the editors October 15, 1964.

1 Research supported by a National Science Foundation grant. 
Observe that $Y_{x}$ is not affected if $X^{\prime}$ is replaced by $\hat{K}$. This is to say the join $O Y_{x}$ of $O$ and $Y_{x}$ is the translation to origin $O$ of the normal cone to $\hat{K}$ at $x$. The set $Y_{0} \subset Y$ is said to be sphere convex if every pair of distinct points in $Y_{0}$ are endpoints of a unique great circle arc contained in $Y_{0}$. It is well known that the normal cone at a boundary point of a convex set is closed and convex. Hence $O Y_{x}$ is closed convex. The use of $R^{n}$ (in place of $R^{N}$ ) assures the absence of antipodal points in $Y_{x}$. Accordingly $Y_{x}$ is closed and sphere convex. It is therefore obviously contractible over $Y$ and so acyclic. (If the $N-1$ unit sphere is used for $Y$, where $N>n$, then $Y_{x}$ need not be acyclic.)

Both $p$ and $q$ are continuous and $p^{-1} y$ is homeomorphic to the acyclic support set $X_{y}$ while $q^{-1} x$, for $x \in X^{\prime}$, is homeomorphic to the acyclic set $Y_{x}$. The Vietoris-Begle Theorem $[1$, p. 503] guarantees therefore that

$$
H_{*}\left(X^{\prime}\right) \approx H_{*}(\Gamma) \approx H_{*}(Y) \text {. }
$$

We remark also that $\hat{K} \cdot$ is of course an $S^{n-1}$. Thus

$$
H_{*}(Y) \approx H_{*}(\hat{K} \cdot) \approx H\left(S^{n-1}\right) .
$$

From (b) and (c) results

$$
H_{*}\left(X^{\prime}\right) \approx H_{*}\left(S^{n-1}\right) .
$$

However since a (closed) proper subset of a topological $n-1$ sphere has a trivial $n-1$ homology group, it follows from (a) that

$$
X^{\prime}=\hat{K}
$$

A Euclidean $n-1$ sphere, $n>1$, is not contractible over a proper subset of the disk it bounds. Accordingly, if $X^{\prime}$, the homeomorph of such a sphere is a proper subset of $X$, then $K$ is a proper subset of $\hat{K}$ and so $X^{\prime}$ is not contractible over $K$ and a fortiori neither is $K$ in contradiction with our hypothesis.

One may conjecture that a similar theorem is valid in a Banach space.

\section{BIBLIOGRAPHY}

1. D. G. Bourgin, Modern algebraic topology, Macmillan, New York, 1963.

2. H. W. Kuhn, Contractibility and convexity, Proc. Amer. Math. Soc. 5 (1954), 777-779.

3. I. Liberman, On certain characteristic properties of convex bodies, Mat. Sb. 13 (55) (1943), 239-262.

UNIVERSITY OF ILLINOIS 